\title{
EFECTO DE LA MUSICOTERAPIA EN EL ESTADO DE ÁNIMO Y CALIDAD DE VIDA DE PACIENTES CON CÁNCER COLORRECTAL
}

\author{
THE EFFECTIVENESS OF MUSIC THERAPY ON MOOD AND QUALITY OF LIFE OF \\ COLORECTAL CANCER PATIENTS
}

\author{
Patricia Martí Augél, Melissa Mercadal-Brotons ${ }^{2}$ y Carme Solé Resano 3 \\ I Oncolliga - Fundació Lliga Catalana d'Ajuda Oncològica, Universitat Ramon Llull. FPCEE Blanquerna \\ 2 Escola Superior de Música de Catalunya (ESMUC) \\ 3 Universitat Ramon Llull. FPCEE Blanquerna
}

Resumen

Objetivo: Evaluar la efectividad de un tratamiento de musicoterapia en el estado anímico y calidad de vida de pacientes diagnosticados de cáncer colorrectal (CCR).

Método: Dieciocho sujetos (14 mujeres y 4 hombres) diagnosticados de CCR participaron en un tratamiento de ocho sesiones de musicoterapia de grupo, de frecuencia semanal y 90 minutos/sesión. Se administró la Escala de Ansiedad y Depresión Hospitalaria (HAD), el Perfil de Estados de Ánimo - versión reducida A (POMS-A) así como la Escala de Calidad de Vida Global y Escalas de Funcionamiento del Cuestionario de Calidad de Vida de la EORTCQLQ-30 (European Organization for Research and Treatment of Cancer-Quality of Life Core Questionnaire-30) en pre- y post-tratamiento. Se midió el nivel de Ansiedad, Tristeza y Malestar Físico mediante una Escala de Valoración Numérica (EVN) en pre- y post-sesión. Se utilizó una Hoja de Observación y Registro de Conductas (instrumento propio -elaborado ad hoc) para registrar las respuestas de cada participante en cada sesión y un Cuestionario de Valoración del Programa de Musicoterapia (instrumento propio, elaborado ad hoc) al finalizar el programa.

Resultados: No se han obtenido cambios estadísticamente significativos en el POMS-A, HAD ni EORTC QLQ-C30, si bien todas las puntuaciones obtenidas van en la dirección
Abstract

Aim: To evaluate the effectiveness of a music therapy treatment on mood and quality of life of colorectal cancer patients.

Method: Eighteen subjects (14 women and 4 men) who were diagnosed with colorectal cancer participated in a music therapy program consisting of eight weekly group music therapy sessions, 90 minute-session. Participants completed the following instruments before and after the intervention (pre/post): the Hospital Anxiety and Depression Scale (HADS), the Profile of Mood States-Short Form A (POMS-A), the Global Quality of Life Scale and the Functioning Scales of the European Organization for Research and Treatment of Cancer-Quality of Life Core QuestionnaireC30 (EORTC-QLQ-C30). A 3 item Numerical Rating Scale (NRS) for Anxiety, Sadness and Physical Distress was administered at the beginning and end of each session (pre/post session). An Observation Behavior Form (designed ad hoc) was used to evaluate mood after each session. A Music Therapy Program Satisfaction Questionnaire (designed ad hoc) was administered at the end of the program.

Results: Although all the obtained scores pointed to a desirable change direction, non significant changes were found in the POMS-A, HADS nor EORTC QLQ-C30. A significant improvement was found in the Anxiety, Sadness and Physical Distress NRS items. The Music 
de cambio deseado. Se ha obtenido una disminución estadísticamente significativa de los niveles de EVN Global de Ansiedad, Tristeza y Malestar. El Cuestionario de Valoración recoge una percepción de alto grado de utilidad y satisfacción por parte de los participantes.

Palabras clave: Cáncer colorrectal, musicoterapia, estado de ánimo, calidad de vida, tratamiento grupal.
Therapy Program Satisfaction Questionnaire showed that participants perceived this type of intervention as very useful and very satisfactory.

Key words: Colorectal cancer, music therapy, mood, quality of life, group treatment.

\section{INTRODUCCIÓN}

El cáncer colorrectal (CCR) es un tipo de cáncer que se presenta en los tejidos del colon (la parte más larga del intestino grueso) o en el recto (los últimos centímetros del intestino grueso). Según el informe de "El Cáncer en España 2014" de la Sociedad Española de Oncología Médica - SEOM ${ }^{(1)}$, el CCR constituye el tercer cáncer más frecuente en hombres (por detrás del de próstata y pulmón), el segundo en mujeres (por detrás del de mama). Si se analiza el CCR en ambos sexos, éste pasa a ser el tipo de cáncer de mayor incidencia (15\%), con una estimación de 32.240 casos (19.261 hombres y 12.979 mujeres). No obstante, estas tasas de incidencia han disminuido en las dos últimas décadas, y este hecho se atribuye al aumento de las pruebas de screening.

La literatura sostiene que los enfermos con cáncer tienen altos niveles de distrés o malestar emocional. La National Comprehensive Cancer Network (NCCN) $)^{(2)}$ desarrolló este término para referirse a esta experiencia emocional desagradable, de naturaleza multifactorial, que incide en la dimensión psicológica, social y/o espiritual de la persona, y que interfiere en la capacidad para afrontar el cáncer de forma efectiva, en sus síntomas físicos y en su tratamiento. Si bien el distrés no es propiamente un trastorno mental, su persistencia en el tiempo podría llevar hacia trastornos mentales diversos. Autores como Hernández y Cruzado ${ }^{(3)}$ sostienen que el sufrimiento psicológico en los enfermos oncológicos llega a alcanzar un nivel de malestar clínico en la mitad de los pacientes, y que puede acompañarse de trastornos psicopatológicos en más del 30\% de los casos. Es por esta razón que se recomienda detectar estos problemas así como derivar a estos pacientes a profesionales de la psicooncología así como de otros ámbitos y especialidades a fin de paliar el malestar emocional y promover una mejor adaptación a la enfermedad, a sus tratamientos y a los posibles efectos secundarios y/o secuelas ${ }^{(4,5)}$.

Los problemas psicológicos que presenta el paciente con CCR pueden venir derivados de la propia enfermedad así como del impacto que los tratamientos tienen en la persona. Tanto el tumor como el tratamiento específico producen secuelas y efectos secundarios que condicionan el funcionamiento físico y psíquico del paciente y obligan a una adaptación y un cambio de comportamiento. En el caso del CCR, algunas personas habrán sufrido una colostomía o ileostomía, y podrán tener problemas psicológicos y sociales de adaptación a la misma. Según Die Trill(6) algunos de los problemas más frecuentes en personas con CCR pueden ser las alteraciones en el estado de ánimo, desarrollo de sentimientos de culpa, alteraciones en la imagen corporal y autoestima, dificulta- 
des en la relación sexual, temor a la pérdida de control así como alteraciones en el nivel de actividad social. Además, las personas afectadas de CCR, al igual que sucede con otros diagnósticos de cáncer, una vez finalizados los tratamientos, han de aprender a convivir con numerosos problemas, adaptarse al estilo de vida resultante y reorganizar su vida. Si bien existen algunos estudios en nuestro país que han empezado a examinar aspectos como la adaptación psicosocial y la calidad de vida de pacientes diagnosticados de CCR, éstos son todavía escasos ${ }^{(7-12)}$.

En un estudio reciente, Cardoso, Blasco y Fernández-Castro ${ }^{(13)}$ revelan que existen poquísimos estudios descriptivos que analicen el afecto positivo o emociones positivas de pacientes con CCR y evidencian la falta de estudios sobre intervenciones psicológicas destinadas a promover emociones y afecto positivo en pacientes diagnosticados de CCR, además de la necesidad de desarrollar estudios de este tipo.

\section{MUSICOTERAPIA Y CÁNCER COLORRECTAL}

La musicoterapia es una disciplina que ha demostrado su eficacia en el contexto oncológico y permite ofrecer un apoyo integral, pudiendo llegar a cubrir necesidades de tipo físico, emocional, cognitivo, social y/o espiritual de la persona ${ }^{(14)}$. Un estudio Cochrane de Bradt, Dileo, Grocke y Magill(15) concluye que un tratamiento con musicoterapia puede aportar resultados beneficiosos en ansiedad, dolor, estado de ánimo y calidad de vida de los pacientes con cáncer y muestra cómo la musicoterapia oncológica es un coadyuvante efectivo y una intervención terapéutica que -ofreciendo un enfoque multimodal e integral- permite cuidar las necesidades del paciente tanto a nivel fisiológico como psicosocial. Actualmente, la musicoterapia está contemplada como una disciplina complementaria y se incluye en el grupo de terapias integrativas utilizadas por entidades como la Society for Integrative Oncology - SIO ${ }^{(16)}$ y el National Center for Complementary and Alternative Medicine - CAM $^{(17)}$. La SEOM ${ }^{(18)}$ ha incluido la musicoterapia dentro de una nueva sección denominada Medicina Integrativa bajo la modalidad de "terapias cuerpo-mente" a fin de que profesionales sanitarios, pacientes y familiares puedan disponer de información basada en la evidencia científica.

Si bien esta disciplina se ha desarroIlado mucho en los últimos 30 años, su aplicación a pacientes con CCR es todavía escasa, a juzgar por los pocos estudios publicados. Existen algunos estudios y meta-análisis llevados a cabo en este contexto que incorporan la música en la fase de diagnóstico con el fin de disminuir los niveles de ansiedad durante pruebas de screening, como colonoscopias o sigmoidoscopias ${ }^{(19-21)}$. No obstante, la mayoría de ellas han sido llevadas a cabo por parte de profesionales sanitarios, muy especialmente por profesionales de enfermería, que corresponderían a un tipo de intervenciones musicales denominadas como "música y medicina", y que no han sido por tanto implementadas por parte de musicoterapeutas profesionales.

Con respecto a la fase de tratamiento, existen interesantes aplicaciones de la música y de la musicoterapia que ofrecen al paciente un apoyo adicional durante tratamientos como cirugía, quimioterapia o radioterapia, entre otros. Si bien se pueden encontrar algunos estudios que han aplicado la música y musicoterapia durante el tratamiento de radioterapia con la finalidad de reducir el distrés emocional de los pacientes con cáncer ${ }^{(22-25)}$ éstos o bien no contemplan específicamente sujetos diagnosticados de CCR o bien van dirigidos a unas muestras que incluyen sujetos con diferentes tipos de cáncer. Asimismo, la literatura ofrece publicaciones centra- 
das en analizar la influencia de la música y musicoterapia durante el tratamiento de quimioterapia, encontrándose ya en este caso un mayor número de experiencias que corresponden a intervenciones de musicoterapia propiamente, y que cuentan por tanto con un musicoterapeuta profesional. Estas intervenciones van dirigidas a reducir náuseas, vómitos y/o otros efectos secundarios, así como para disminuir la ansiedad y/o malestar emocional y mejorar el estado de ánimo, promover estados de relajación, mejorar variables fisiológicas y/o funcionamiento físico ${ }^{(26-32)}$ así como para observar el efecto en la calidad de vida $^{(33-37)}$. No obstante, siguen sin encontrarse fácilmente estudios dirigidos a una muestra homogénea de sujetos diagnosticados de CCR exclusivamente. Una interesante aplicación de esta disciplina se da en el contexto de fase paliativa, y va dirigida a mejorar la calidad de vida, a través de la reducción de síntomas de malestar físico, cubrir las necesidades psicológicas, ofrecer soporte, facilitar la comunicación y cubrir las necesidades espirituales ${ }^{(38)}$. En estos casos, se trata también de estudios que incorporan sujetos con diagnósticos oncológicos heterogéneos.

La literatura indica, pues, que los pacientes con cáncer experimentan elevados niveles de distrés emocional que repercuten en su salud y que la musicoterapia ha mostrado ser una intervención eficaz para paliar ese distrés, y mejorar el estado anímico y la calidad de vida de los enfermos con cáncer. Sin embargo, esta disciplina tiene todavía mucho camino por recorrer para diseñar y desarrollar nuevos estudios y programas de musicoterapia aplicada al CCR e implementados por parte de musicoterapeutas profesionales.

Los objetivos específicos de este estudio fueron:

1. Evaluar si hay una mejora del estado anímico, ansiedad, depresión y calidad de vida de un grupo de pacientes diagnosticados de CCR una vez finalizado el tratamiento de 8 sesiones de musicoterapia (pre-/post-tratamiento).

2. Evaluar si hay una mejora en el estado percibido de ansiedad, tristeza y malestar físico después de cada sesión de musicoterapia (pre/post-sesión).

\section{MÉTODO}

\section{Muestra}

Los criterios de inclusión para el estudio fueron los siguientes: a) sujetos adultos, mayores de 18 años, diagnosticados de cáncer CCR; b) estar en tratamiento oncológico activo (quimioterapia, radioterapia, o pendientes de cirugía) o haberlo finalizado en los últimos 3 meses antes de iniciar la primera sesión de musicoterapia; c) presencia de malestar emocional; d) no haber recibido previamente un tratamiento de musicoterapia; y e) haber dado su consentimiento para participar en este estudio. Para el análisis estadístico se estableció un criterio de inclusión adicional: haber asistido como mínimo al $50 \%$ de las sesiones. Los criterios de exclusión fueron: a) presencia de patología psiquiátrica previa; b) problemas de hipoacusia moderada o severa; y c) dificultad para desplazarse semanalmente al hospital.

La muestra final de sujetos estuvo compuesta por un total de 18 sujetos, 14 mujeres y 4 hombres, con un rango de edad es de 31-79 años $(M=64,33, D T=13,19)$. El perfil clínico de los participantes se caracteriza por ser un grupo de sujetos diagnosticados de cáncer de colon $(n=13)$ y recto $(n=5)$ en diferentes estadios de la enfermedad. La mayoría de ellos fueron diagnosticados de cáncer entre 7 y 18 meses antes de iniciar el tratamiento de musicoterapia; 11 participantes estaban recibiendo tratamiento de quimioterapia, 
mientras que el resto no se encontraba en ese momento recibiendo ningún tratamiento, bien porque acababa de finalizar el tratamiento oncológico activo $(n=4)$ o por estar a la espera de intervención quirúrgica $(\mathrm{n}=3)$. La tabla 1 recoge los datos sociodemográficos y clínicos más relevantes de esta muestra.

\section{Tabla 1. Datos Demográficos y Clínicos de la Muestra ( $\mathbf{N}=\mathbf{1 8})$}

\begin{tabular}{|c|c|}
\hline Variables Demográficas & \\
\hline Sexo & $\mathrm{n}$ \\
\hline Mujeres & 14 \\
\hline Hombres & 4 \\
\hline Edad & $31-79$ años $(M=64,33 ; D T=13,195)$ \\
\hline Estado Civil & $\mathrm{n}$ \\
\hline Soltero & 4 \\
\hline Casado/a / o en pareja & 7 \\
\hline Separado/a & 2 \\
\hline Viudo/a & 5 \\
\hline $\mathrm{N}^{o}$ de Hijos & $\mathrm{n}$ \\
\hline Ninguno & 7 \\
\hline 1 & 3 \\
\hline 2 & 3 \\
\hline 3 o más & 7 \\
\hline Nivel de Estudios & $\mathrm{n}$ \\
\hline Primarios & 8 \\
\hline Secundarios & 5 \\
\hline Superiores & 5 \\
\hline Situación Ocupacional Actual & $\mathrm{n}$ \\
\hline De baja & 9 \\
\hline Jubilado & 9 \\
\hline Activo & 0 \\
\hline Variables Clínicas & \\
\hline Diagnóstico & $\mathrm{n}$ \\
\hline Colon & 13 \\
\hline Recto & 5 \\
\hline Periodo de Tiempo desde el Momento del Diagnóstico & $\mathrm{n}$ \\
\hline Menos de 6 meses & 1 \\
\hline Entre 7 y 12 meses & 10 \\
\hline Entre 13 y 18 meses & 6 \\
\hline Más de 19 meses & 1 \\
\hline Tratamientos Previos & $\mathrm{n}$ \\
\hline Cirugía & 5 \\
\hline Quimioterapia & 3 \\
\hline Quimioterapia+Cirugía & 2 \\
\hline Quimioterapia+Radioterapia & 4 \\
\hline Quimioterapia+Cirugía+Radioterapia & 4 \\
\hline Tratamiento Médico Actual & $\mathrm{n}$ \\
\hline En espera de cirugía & 3 \\
\hline Quimioterapia & 11 \\
\hline Ninguno & 4 \\
\hline Soporte emocional & $\mathrm{n}$ \\
\hline Sí & 1 \\
\hline No & 17 \\
\hline Medicación psicofármacos & $\mathrm{n}$ \\
\hline Sí & 3 \\
\hline No & 15 \\
\hline
\end{tabular}


Instrumentos de evaluación

Se trata de un diseño intrasujeto, de medidas repetidas. Se utilizaron los siguientes instrumentos:

- El Pefil de Estados de Ánimo - Abreviado $(P O M S)^{(39)}$ consta de 58 ítems que permite evaluar el estado de ánimo. Para este estudio, con el objetivo de facilitar su aplicación a las pacientes, se utilizó el POMS-A, la versión reducida A de 15 ítems elaborada por Fuentes, García-Merita, Melià y Balaguer ${ }^{(40)}$. Se trata de un auto-informe que incluye 4 escalas de afectos negativos, Tensión, Depresión, Cólera y Fatiga, y una escala de afecto positivo, Vigor. Consta de 15 ítems, que son adjetivos sobre diferentes estados afectivos de los que el paciente ha de graduar la intensidad de cada uno de ellos según los haya sentido durante los últimos 7 días, y en un formato de escala Likert de 5 puntos. También ha sido frecuentemente utilizada en el campo de la oncología así como de la musicoterapia.

- La Escala Hospitalaria de Ansiedad y Depresión, HAD (Hospital Anxiety and Depression Scale)(41) es una escala ampliamente utilizada en oncología, que evalúa la presencia de síntomas de ansiedad y depresión en pacientes con enfermedad física en el contexto hospitalario. Tiene un total de 14 ítems, repartidos en dos subescalas de 7 ítems cada una, para medir: Ansiedad y Depresión. Consta de 4 opciones relativas a la frecuencia con la que se da un cierto tipo de respuesta definida como de ansiedad o de depresión, en un marco temporal de los últimos 7 días. El rango de puntuación es de 0-21 para cada subescala y de 0-42 para la puntuación global. Para este estudio se utilizó la versión española, adaptada de Caro e Ibáñez ${ }^{(42)}$.

- El cuestionario de Calidad de Vida de la EORTC QLQ-C30 (European Organiza- tion for Research and Treatment of Cancer-Quality of Life Core Questionnai$r \mathrm{e}^{(43)}$ es un instrumento para evaluar de manera específica la calidad de vida en sus diferentes dimensiones en pacientes con cáncer. Consta de 30 ítems, 28 de ellos con 4 alternativas de respuesta y 2 de ellos con 7 alternativas (donde 1 es pésima y 7 excelente), y el marco temporal se refiere a la semana previa. Está compuesto de 3 escalas: Estatus de Salud Global, Escalas Funcionales, y Escala de Síntomas. Para este estudio se ha utilizado la versión española de Arrarás, Illarramendi y Valerdi ${ }^{(44)}$, y se han utilizado la Escala Global de Calidad de Vida y las 5 Escalas de Funcionamiento. Si bien existe un módulo específico que complementa el cuestionario original y va dirigido a pacientes con cáncer colorrectal (EORTC QLQCR29) y que ha sido también validado en nuestro país ${ }^{(45)}$, se desestimó su administración por dos razones: por un lado, por una cuestión de practicidad, ya que el test se rellena en el mismo momento antes de iniciar la sesión y requeriría alargar aún más el tiempo de administración, cuando las dificultades de concentración suelen ser un síntoma frecuente en enfermos en tratamiento oncológico activo, y por otro lado, por respeto a los pacientes, a fin de no resultar demasiado invasivos con preguntas y cuestiones de carácter muy íntimo. Es un test habitualmente utilizado en el campo de la musicoterapia.

- La Escala de Valoración Numérica (EVN) es una escala empleada habitualmente en el contexto hospitalario como un instrumento rápido y sencillo de screening, que tiene como objetivo evaluar la intensidad de un síntoma. En este estudio se utilizó una ENV de 11 puntos (0-10), en la que se presenta una línea recta horizontal numerada de 0 a 10, en cuyos extremos se repre- 
senta la polarización de la intensidad del síntoma (donde 0 es la ausencia del síntoma y 10 el síntoma a un nivel máximo e insoportable). Se han presentado 3 EVN para los 3 ítems elegidos: Ansiedad, Tristeza y Malestar Físico, y se pide a los participantes que las completen al inicio y final de cada sesión. Las EVN miden el cambio tras 90 minutos de haber participado en una sesión de musicoterapia.

- Hoja de Observación y Registro de Conductas (instrumento diseñado ad hoc): Fue utilizada y cumplimentada por la musicoterapeuta para evaluar el estado anímico, después de cada sesión a fin de recoger las respuestas de cada participante sobre aspectos relacionados con su expresión emocional, atención-participación en la tarea musical, interacción-comunicación social, y asistencia a la sesión.

- Cuestionario de Valoración del Programa de Musicoterapia (instrumento diseñado ad hoc): una vez finalizado el programa, se pidió a los participantes que rellenaran este cuestionario de manera anónima, para proporcionar feedback e información sobre sus experiencias en las sesiones de musicoterapia.

\section{Procedimiento}

El proyecto de este estudio fue presentado al equipo de Oncología Médica del Hospital de la Santa Creu i Sant Pau de Barcelona y al Comité de Ética e Investigación (CEIC). Tras su aprobación se procedió a coordinar el proyecto con los oncólogos especialistas en cáncer colorrectal. Dos oncólogos de este equipo fueron los encargados de reclutar a los pacientes para este estudio. Aquellos pacientes que cumplían con los criterios de inclusión eran directamente informados por su oncólogo de referencia sobre la existencia de esta investigación, e invitados a participar en la misma. Si el sujeto mostraba interés por participar, se le incluía en una lista de espera. Una vez configurado un grupo de entre 8-10 personas, se iniciaba cada programa de tratamiento. Los participantes leyeron una Hoja de Información del Estudio y firmaron el documento de Consentimiento Informado antes de empezar a participar en musicoterapia.

Antes de iniciar el tratamiento (antes de la sesión 1), se procedió a la administración de los instrumentos de evaluación que se han descrito anteriormente. Antes y después de cada sesión, se solicitaba a cada participante que rellenase la Escala de Valoración Numérica para cada uno de los 3 ítems. Después de cada sesión, la musicoterapeuta cumplimentaba, para cada paciente, la Hoja de Observación y Registro de Conductas observadas en la sesión. Una vez finalizado el tratamiento, se volvieron a aplicar los mismos instrumentos de evaluación, juntamente con el Cuestionario de Valoración del Programa de Musicoterapia.

Semanalmente, se implementaba una sesión de musicoterapia de grupo de 90 minutos de duración, en una de las salas del hospital adjudicada temporalmente para este proyecto. A lo largo de todo el estudio, se configuraron 5 grupos en total; una vez finalizaba un grupo se procedía a iniciar el siguiente.

\section{Sesiones de Musicoterapia}

Todas las sesiones de musicoterapia fueron dirigidas por la misma musicoterapeuta profesional, especializada a su vez en psicooncología. La intervención de musicoterapia consistió en la aplicación de diferentes técnicas y actividades musicales, siempre diseñadas e implementadas para trabajar objetivos terapéuticos dirigidos a favorecer la expresión y ventilación emocional, a canalizar emociones y sentimientos a nivel verbal y no verbal mediante la música, a facilitar estados de relajación y 
distracción así como a promover la interacción grupal. Los enfermos oncológicos deben vivir situaciones que generan mucha incertidumbre con respecto a su condición física, a los tratamientos y a la evolución y curso de la enfermedad, entre otros aspectos. Es recomendable ofrecer un espacio terapéutico estructurado, que aporte seguridad y predictibilidad. Es por esta razón que este programa siguió la misma estructura en cada sesión: se iniciaba con una actividad de apertura, se desarrollaba mediante la implementación de diferentes técnicas y actividades relacionadas con el tema a abordar en cada sesión, y finalizaba con una actividad de cierre. Se combinaron técnicas de carácter activo (cantar canciones, análisis lírico, tocar instrumentos musicales, improvisación instrumental, música y movimiento, entre otras) con técnicas de tipo pasivo-receptivo (relajación acompañada de música y audiciones musicales).

\section{Análisis estadístico}

El análisis cuantitativo se ha realizado calculando los estadísticos descriptivos y utilizando la prueba $t$ de Student, una prueba paramétrica que sigue una distribución normal pero se ajusta al tamaño pequeño de la muestra, a fin de comparar los resultados entre el pre- $y$ el post-tratamiento de los tests POMS-A, HADS, y EORTC QLQ-C30. También se aplicó esta prueba para comparar los resultados pre- y postsesión de las puntuaciones de cada una de las tres EVN (Ansiedad, Tristeza y Malestar Físico). Dado el tamaño pequeño de la muestra, se utilizó la corrección HolmBonferroni para contrarrestar el problema de las múltiples comparaciones, y reducir la probabilidad de cometer error de tipo 1 debido a la gran cantidad de hipótesis, o problema de la multiplicidad $(p=0,05 / n)$. Se partió de un nivel de significación de $p$ $\leq 0,05$. Adicionalmente, se utilizó el test de Cohen $d$ para evaluar el tamaño del efecto de aquellos valores significativos. Para llevar a cabo el análisis estadístico se empleó el programa estadístico IBM SPSS Statistics versión 21.

\section{RESULTADOS}

De los 25 sujetos que acabaron participando en el estudio, 7 de ellos tuvieron que ser excluidos del análisis final por no haber podido completar los post-tests o por haber faltado a más del $50 \%$ de las sesiones (a fin de poder valorar de una manera más rigurosa la eficacia de la intervención, los investigadores establecieron como criterio adicional de inclusión para el análisis de datos el haber asistido al menos al 50\% de las sesiones). Por todo ello, la muestra final fue de 18 pacientes. El promedio de asistencia fue de 6,16 sobre 8 sesiones, lo que equivaldría a un $77,08 \%$ del total de sesiones. Un $22,22 \%(n=4)$ de los pacientes asistió al $100 \%$ del tratamiento (8 sesiones en total); un $16,66 \%(n=3)$ lo hizo al $87,50 \%$ (7 sesiones); el 33,33\% $(n=6)$ corresponde a aquellos participantes que han asistido al $75 \%$ del tratamiento $(6$ sesiones); el $11,11 \%(n=2)$ asistió al $62,50 \%$ del tratamiento (5 sesiones); y el $16,66 \%$ $(n=3)$ lo hizo al 50\% del mismo (4 sesiones). El análisis dosis-resultados no ha sido estadísticamente significativo para ninguna de los tests empleados, de modo que no se ha podido demostrar una relación directa entre una mayor frecuencia de asistencia y un mejor resultado.

Los resultados para cada variable dependiente son los siguientes:

\section{Análisis cuantitativo}

Estado de Ánimo, Ansiedad, y Depresión (cambios pre- y post-tratamiento):

La prueba $t$ de Student ha permitido comparar la media de las diferencias entre las puntuaciones medias del pre- y posttratamiento para los niveles de Tensión, 
Depresión, Cólera, Vigor y Fatiga así como del Índice de Alteración Emocional (IAE) de la escala POMS-A. Ninguna de ellas muestra diferencia significativa. No obstante, y a excepción de la subescala Vigor, todas las puntuaciones disminuyen al finalizar el tratamiento. La subescala Vigor muestra una puntuación más elevada en el post-tratamiento, un aumento que en este caso representa una "mejora" del síntoma dado que se trata de una variable positiva (ver tabla 2).

Con respecto a la media de puntuaciones pre-tratamiento de cada subescala, se observa que todas ellas se sitúan en un rango bajo de malestar emocional: Tensión $M=3,83$ ( $D T=2,640)$, Depresión $M=3,11$ ( $\mathrm{DT}=2,139)$, Cólera $M=2,83$ (DT=2,503), Vigor $M=4,78(D T=2,157)$, y Fatiga $M=$ 4,44 (DT= 3,276). La media de puntuaciones obtenidas en el post-tratamiento se mantienen en este rango bajo de malestar emocional: Tensión $M=3,11$ (DT=2,246), Depresión $M=1,72(\mathrm{DT}=1,965)$, Cólera $M=2,11$ (DT=2,298), Vigor $M=5,50$ (DT= $2,728)$, y Fatiga $M=3,44$ (DT=2,617). El IAE también presenta unas puntuaciones que corresponden a un rango de bajo malestar emocional, con una media de $M=$ $21,44(\mathrm{DT}=8,900)$ antes de iniciar el tratamiento que se reduce a $17,11(\mathrm{DT}=8,415)$ tras finalizar el tratamiento.

El análisis de las diferencias entre las medias de las puntuaciones pre- y post-tratamiento para los niveles de Ansiedad y de Depresión de la escala HAD señala que, si bien estas puntuaciones disminuyen para ambas subescalas, éstas no llegan a mostrar diferencias significativas (ver tabla 3).

Tanto las puntuaciones obtenidas en el total de la muestra para la variable Ansiedad como para la variable Depresión se encuentran dentro de la normalidad, y no muestran patología clínica ni antes ni des-

\section{Tabla 2. Índice de Alteración Emocional y Subescalas del POMS-A: Media de las Puntuaciones Pre/Post-Tratamiento, Desviación Tipo, t de Student, Grados de Libertad y Valor de Significación Bilateral}

\begin{tabular}{lccccc}
\hline Subescala & Media & DT & $\mathrm{t}$ & $\mathrm{gl}$ & Sig. (bilateral) \\
\hline $\begin{array}{l}\text { Índice Alteración Emocional } \\
\text { POM-S }\end{array}$ & 4,33 & 8,430 & 2,181 & 17 & 0,044 \\
Tensión & 0,72 & 1,674 & 1,831 & 17 & 0,085 \\
Depresión & 1,38 & 2,062 & 2,858 & 17 & 0,011 \\
Cólera & 0,72 & 2,347 & 1,306 & 17 & 0,209 \\
Vigor & $-0,72$ & 2,396 & $-1,279$ & 17 & 0,218 \\
Fatiga & 1,00 & 3,029 & 1,401 & 17 & 0,179 \\
\hline
\end{tabular}

Valores-p de corrección de Holm Bonferroni: 0,008; 0,01; 0,0125; 0,016; 0,025 y 0,05.

Tabla 3. HADS: Media de las Puntuaciones Pre/Post-Tratamiento, Desviación Tipo, t de Student, Grados de Libertad y Valor de Significación Bilateral

\begin{tabular}{lccccc}
\hline Subescala & Media & DT & $\mathrm{t}$ & $\mathrm{gl}$ & Sig. (bilateral) \\
\hline Ansiedad & 0,94 & 2,287 & 1,752 & 17 & 0,098 \\
Depresión & 0,61 & 2,638 & 0,983 & 17 & 0,339 \\
\hline
\end{tabular}

Valores-p de corrección de Holm Bonferroni: 0,025 y 0,05. 
pués del tratamiento. Las puntuaciones obtenidas en la subescala Ansiedad son más elevadas que en las de Depresión. Así, los pacientes con CCR mostraron en el pretratamiento una media de 7,83 sobre 21 puntos (DT=3,944) en la variable Ansiedad. Esta puntuación se ubica dentro del rango de normalidad, aunque ligeramente por debajo del rango de un estado subclínico. Al finalizar el tratamiento, esta puntuación pasa a ser de $M=6,89$ ( $D T=3,341$ ). De los cuatro sujetos que presentaban un estado de ansiedad clínica en el pre-tratamiento, hay un sujeto que evolucionó a un estado de normalidad y otro a un estado subclínico; los otros dos se han mantenido como casos clínicos. De los tres sujetos que presentaban ansiedad a nivel subclínico, uno de ellos pasó a un estado normal, otro alcanzó un resultado clínico y el otro se mantuvo igual. Los 11 sujetos restantes comenzaron el tratamiento en un estado normal y se mantuvieron estables a excepción de dos de ellos que pasaron a ser calificados como un caso subclínico.

En cuanto a la variable Depresión, la media fue de $M=4,56$ sobre 21 puntos (DT $=3,034)$ en el pre-tratamiento, una puntuación que se ubica dentro del rango de normalidad. Una vez finalizado el tratamiento, esta media se redujo a $M=3,94$ (DT=2,980). En el momento del pre-tratamiento, únicamente tres sujetos presentaban un estado subclínico, de los cuales dos de ellos pasaron a un estado normal al finalizar el tratamiento mientras que el otro se mantuvo igual. El resto de sujetos partieron ya de un estado normal al iniciar el tratamiento, pasando dos de ellos al estado subclínico al finalizar éste.

Ansiedad, Tristeza y Malestar Físico (cambios pre- y post-sesión):

Se ha aplicado la prueba $t$ de Student para analizar el efecto de la intervención, comparando, para cada uno de los tres ítems, la media global de todas las puntuaciones medias de EVN a nivel pre-sesión (sesión 1 a la 8 pre-) con la media global de todas las puntuaciones medias de EVN a nivel post-sesión (sesión 1 a la 8 post-). Los resultados extraídos de esta prueba muestran una disminución estadísticamente significativa de los niveles de Ansiedad, Tristeza y Malestar Físico. La corrección Holm-Bonferroni para múltiples comparaciones mostró diferencias significativas para el ítem Ansiedad (valor-p=0,016), para el ítem Malestar Físico (valor-p=0,025), y para el de Tristeza (valor-p=0,05). El cálculo de la $d$ de Cohen muestra un tamaño del efecto grande para los tres casos, destacando especialmente el Malestar Físico. La tabla 4 recoge estos resultados.

Los estadísticos descriptivos de la EVN ofrecen unas puntuaciones bajas. La media de la variable Ansiedad de la EVN fue de $M=1,91 \quad(D T=2,007)$ en el momento de pre-sesión se redujo a $\mathrm{M}=0,87(\mathrm{DT}=0,952)$

\section{Tabla 4. EVN de Ansiedad, Tristeza y Malestar Físico: Media de la Puntuaciones Pre/Post-Sesión, Desviación Tipo, t de Student, Grados de Libertad, Valor de Significación Bilateral y d de Cohen}

\begin{tabular}{lcccccc}
\hline Subescala & Media & DT & t & gl & Sig. (bilateral) & d \\
\hline Ansiedad & 1,03 & 1,204 & 3,642 & 17 & $0,002^{* *}$ & 0,85 \\
Tristeza & 0,93 & 1,156 & 3,421 & 17 & $0,003^{* * *}$ & 0,80 \\
Malestar Físico & 0,85 & 0,676 & 5,345 & 17 & $0,000^{*}$ & 1,25 \\
\hline
\end{tabular}

Valores-p de corrección de Holm Bonferroni: * $p \leq 0,016(0,05 / 3)$; ${ }^{* *} p \leq 0,025(0,05 / 2) ;{ }^{* * *} p \leq 0,05$. 
en el post-sesión. La variable Tristeza pasa de una media inicial de $M=1,82(\mathrm{DT}=1,687)$ a una media final de $M=0,88(D T=0,890)$; y finalmente, la variable Malestar Físico tiene una media inicial de $M=1,8(D T=1,206)$ que disminuye a una $M=1,00(\mathrm{DT}=0,811)$ después de finalizar la sesión.

Calidad de Vida (cambios pre- y post-tratamiento)

Este estudio ha analizado la Calidad de Vida a partir de las siguientes dimensiones del cuestionario de Calidad de Vida de la EORTC QLQ-C30: la Escala de Calidad de Vida Global y las cinco Escalas de Funcionamiento (F). No se han administrado las 3 Escalas de Síntomas. Los resultados muestran unas puntuaciones negativas obtenidas de la $t$ de Student, lo que indica que la puntuación pre-tratamiento es inferior a la post-tratamiento en la Escala Calidad de Vida Global así como para las 5 subescalas de Funcionamiento. Este hecho debe ser interpretado como una mejora, dado que se trata de escalas positivas, y una puntuación mayor corresponde a una mayor y mejor calidad de vida. No obstante, pese a esta mejora, los resultados no llegan a alcanzar significación estadística en ninguna escala ni subescala. La sub- escala de Funcionamiento Emocional es la que parte de un nivel inicial más bajo y la que, al finalizar el tratamiento, consigue, una diferencia mayor y, por tanto, una puntuación más elevada que todas las demás (ver tabla 5).

Los estadísticos de cada una de las puntuaciones pre- y post-tratamiento muestran los siguientes resultados: Calidad de Vida Global pre- $M=60,66(\mathrm{DT}=20,595)$ y post- $M=67,12$ (DT= 18,845); F. Físico pre$M=84,56(\mathrm{DT}=13,183)$ y post- $M=87,56$ $(\mathrm{DT}=15,779) ; \mathrm{F}$. de Rol pre- $\mathrm{M}=69,67$ (DT= $29,766)$ y post- $M=72,44$ (DT= 31,260); F. Emocional pre- $M=62,72(\mathrm{DT}=19,637)$ y post- $M=71,94$ (DT= 16,728); F. Cognitivo pre- $M=79,94$ (DT= 20,174) y post$M=80,83(\mathrm{DT}=16,332) ; \mathrm{F}$. Social pre- $\mathrm{M}=$ $77,11(\mathrm{DT}=19,778)$ y post- $\mathrm{M}=81,78(\mathrm{DT}=$ $15,973)$.

\section{Análisis cualitativo}

Resultados Cuestionario de Valoración del Programa de Musicoterapia

Este cuestionario, diseñado ad hoc, ha permitido explorar varios aspectos. Con respecto a la percepción de la mejora del estado anímico durante las sesiones, 13 de los participantes expresan que este pro-

\section{Tabla 5. EORTC-QLQ-C30 Calidad de Vida: Media de las Puntuaciones Pre/Post- Tratamiento, Desviación Tipo y Valores de Significación}

\begin{tabular}{lccccc}
\hline Escala & Media & DT & $\mathrm{t}$ & $\mathrm{gl}$ & Sig. (bilateral) \\
\hline Calidad de Vida Global (a) & $-6,46$ & 20,128 & $-1,362$ & 17 & 0,191 \\
${\text { Funcionamiento: }{ }^{(b)}}$ & & & & \\
- Funcionamiento Físico & $-3,00$ & 11,040 & $-1,153$ & 17 & 0,265 \\
- Funcionamiento de Rol & $-2,77$ & 22,918 & $-0,514$ & 17 & 0,614 \\
- Funcionamiento Emocional & $-9,22$ & 21,195 & $-1,846$ & 17 & 0,082 \\
- Funcionamiento Cognitivo & $-0,88$ & 16,684 & $-0,226$ & 17 & 0,824 \\
- Funcionamiento Social & $-4,66$ & 18,818 & $-1,052$ & 17 & 0,307 \\
\hline
\end{tabular}

(a) Valores-p de corrección de Holm Bonferroni: 0,05

(b) Valores-p de corrección de Holm Bonferroni: 0,01; 0,0125; 0,016; 0,025 y 0,05 
grama les ha ayudado a mejorar "Mucho" su estado anímico, mientras que los otros cinco consideran que esta mejora ha sido "Bastante". Esta percepción de ayuda disminuye algo más cuando se trata de puntuar sobre la mejora del estado anímico en el día a día ( $y$, por tanto, en situaciones y momentos fuera de la sesión) en cuanto a Ansiedad y a Tristeza. Aún y así, los resultados obtenidos siguen siendo positivos (ver figura 1).
El cuestionario ha permitido también explorar cómo los participantes han valorado las diferentes estrategias de autorregulación emocional: la expresión de emociones y sentimientos, hablar de la enfermedad, la distracción y evasión de las preocupaciones, la relajación y disminución de tensiones, así como la interacción con los compañeros del grupo (socialización). La figura 2 muestra los resultados obtenidos para cada una de ellas.

Figura 1. Percepción de Mejora del Estado Anímico

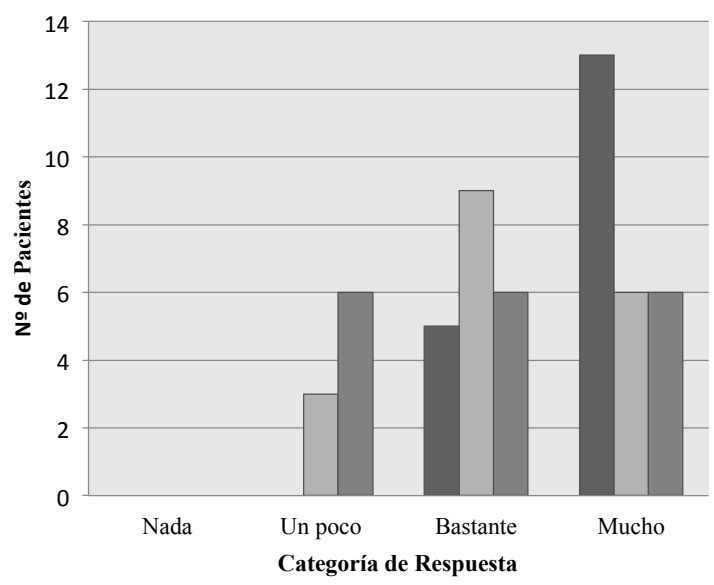

- Mejorar estado anímico durante sesión

$\square$ Ayuda a disminuir ansiedad día a día

$\square$ Ayuda a disminuir tristeza día a día

Figura 2. Percepción sobre las Estrategias de Autorregulación Emocional

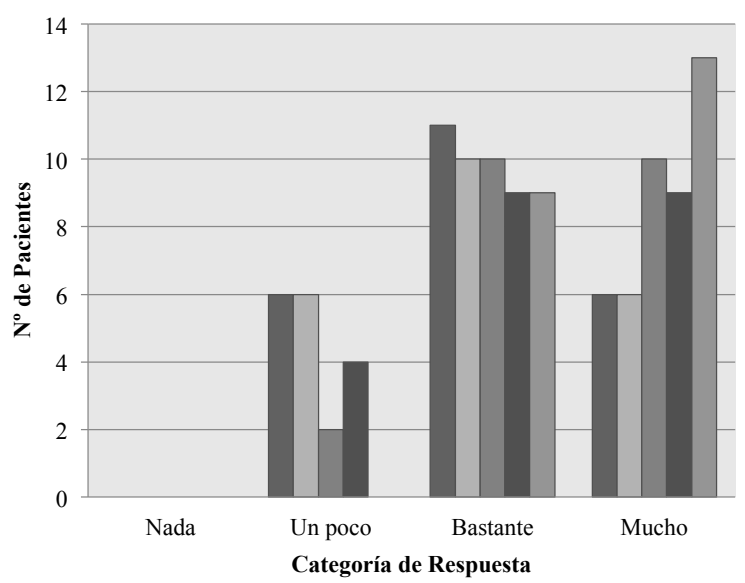

Expresión emocional

$\square$ Hablar de la enfermedad

$\square$ Distracción y evasión

- Relajación

$\square$ Socialización 
En general, se puede afirmar que los participantes han percibido que este programa ha promovido el aprendizaje de recursos de auto-cuidado de los participantes, ya que lo han valorado como "Mucho" ( $n=12)$, "Bastante $(n=5)$ y "Un poco" $(n=1)$. La percepción del grado de utilidad de este programa ha sido alta, en tanto que 12 sujetos consideran que esta experiencia ha resultado muy útil ("Mucho"), para cinco de ellos ha sido "Bastante" útil, mientras que para uno de ellos sólo "Un poco". Finalmente destacar el hecho de que los participantes coinciden en que esta experiencia es recomendable para otras personas: "Mucho" $(n=12)$ y "Bastante" $(n=6)$.

\section{DISCUSIÓN}

El objetivo de este estudio se ha centrado en explorar la eficacia de un tratamiento con musicoterapia para mejorar el estado anímico y calidad de vida de un grupo de pacientes diagnosticados de cáncer colorrectal. Con respecto al análisis cuantitativo del estado anímico, se comentan seguidamente los resultados obtenidos a nivel de post-tratamiento para las variables estado de ánimo, ansiedad y depresión.

Los resultados de la escala POMS-A no muestran diferencia significativa en ninguna de sus cinco subescalas ni en la del Índice de Alteración Emocional (IAE). No obstante, sí se debe destacar el hecho de que todas las puntuaciones, salvo las de la subescala Vigor, muestran una disminución, hecho que va en la línea de los cambios esperados. La subescala Vigor, a diferencia de las otras subescalas de carácter negativo de este test (Tensión, Depresión, Cólera y Fatiga), es una variable positiva, de modo que su puntuación más elevada en el post-tratamiento representa una mejora del síntoma. El IAE muestra una disminución de la puntuación que tampoco no resulta significativa estadísticamente. La no significación de estos datos podría explicarse por el hecho de que esta muestra presenta un rango bajo de malestar y alteración emocional ya antes de iniciar el tratamiento y por tanto hay poca posibilidad de cambio positivo o mejora. Ante una falta de resultados significativos en el IAE de su estudio, Waldon ${ }^{(46)}$ argumentó como posible causa el hecho de que los estados de ánimo son transitorios y fluctuantes por naturaleza, y que el POMS es un instrumento sensible a estos cambios del estado anímico, sin detectar propiamente un rasgo que persista de manera más estable en el tiempo. Otra posible explicación que podría ayudar a entender la falta de significación en este estudio sería el hecho de que la versión reducida $\mathrm{A}$ de este test no haya sido suficientemente sensible para recoger todo el malestar emocional de los participantes. Además, la prueba estadística de Holm-Bonferroni conlleva realizar un ajuste del valor-p en función del número de contrastes, que en el caso del POMSA es de 6, lo que lleva a establecer un primer nivel $\alpha$ de significación de $p=0,011$ $(0,05 / 6)$. No obstante, los resultados obtenidos con este test van en la dirección de cambio deseado, y apuntan hacia los resultados obtenidos en estudios previos realizados, en los que se logran unas mejoras significativas del estado de ánimo como resultado de participar en sesiones de musicoterapia en el contexto ${ }^{(46-48)}$.

La subescala de Depresión es la que presenta un valor $p$ más bajo de todas $(p=$ 0,011 ) seguida del índice de Alteración Emocional $(p=0,044)$ y de la subescala Tensión $(p=0,85)$. Un dato interesante a comentar es el hecho de que la puntuación más baja de todas las subescalas corresponde a la subescala Depresión, que aunque no alcanza nivel de significación estadística, contrasta con los resultados de la revisión Cochrane de Bradt et al. ${ }^{(15)}$ que sugieren que las variables depresión o fatiga de los pacientes con cáncer no responden de manera tan clara ante las inter- 
venciones con musicoterapia, como sí suele hacerlo la variable ansiedad (Tensión). Otro dato a comentar es la congruencia de los resultados obtenidos: las subescalas Depresión y Tensión (Ansiedad) quedan más próximas al grado de significación que las otras subescalas. Ello puede explicarse porque este programa ha incidido especial y directamente en la variable Depresión (y Tristeza) y Ansiedad (Tensión) mediante técnicas y actividades de musicoterapia específicamente diseñadas para promover una mejora en estos dos ítems del estado anímico (y no ha pretendido incidir directamente en variables como Cólera, Fatiga y Vigor, si bien ha obtenido resultados en la dirección deseada y que apuntan, por tanto, hacia una mejora).

Los resultados de la escala HAD tampoco reflejan un cambio significativo en sus puntuaciones de sintomatología ansiosodepresiva, si bien también se da una disminución en las mismas. Tanto las puntuaciones obtenidas en el total de la muestra para la variable Ansiedad como para la variable Depresión se encuentran dentro de la normalidad, y no muestran patología clínica ni antes ni después del tratamiento. Las puntuaciones obtenidas en la subescala Ansiedad en el pre-tratamiento se ubican ligeramente por debajo del rango de un estado subclínico, y disminuyen al finalizar el tratamiento. La mayor parte de los participantes se sitúan en un rango de normalidad; solo un 22,22\% ( $n=4)$ de la muestra presenta problemas de ansiedad clínica, y la mitad de ellos reduce este malestar al acabar el tratamiento; el otro 16,66\% $(n=3)$ presentaba en un inicio un estado de ansiedad a nivel subclínico, que mostró variabilidad en los resultados, mejorando a un estado normal, empeorando a un estado clínico y manteniéndose igual. Los 11 sujetos restantes comenzaron el tratamiento en un estado normal y se mantuvieron estables a excepción de dos de ellos que pasaron a ser calificados como un caso subclínico.
La variable Depresión obtuvo puntuaciones muy bajas en el pre-y post-tratamiento, que se consideran dentro del rango de normalidad. No se detectaron casos de depresión clínica en el pre-tratamiento, y únicamente el $16,66 \%(n=3)$ de los sujetos presentaban un estado subclínico, que al finalizar el tratamiento progresaron a un estado normal $(n=2)$ y manteniéndose igual en el otro caso $(n=1)$. Tan solo un $11,11 \%$ finalizaron el tratamiento en el rango de depresión subclínica, mientras lo habían iniciado en rango de normalidad.

Como conclusión, cabe señalar que, a nivel general, tanto la variable Ansiedad como Depresión del HAD presentan puntuaciones que ya en el se sitúan en un rango de normalidad, de no patología clínica, y disminuyen un poco tras el tratamiento. Esta muestra presenta, pues, unos niveles de distrés emocional (ansiedad y depresión) bajos. Estos resultados coinciden con las puntuaciones también bajas obtenidas en el test POMS-A comentado anteriormente. Así pues, en el caso de esta muestra, no parece haber presencia de sintomatología a la que tratar con esta intervención, lo que podría explicar también la no obtención de cambios significativos en los resultados.

Continuando con el análisis cuantitativo, se comentan a continuación aquellos resultados obtenidos en pre- y post-sesión para las variables ansiedad, tristeza y malestar físico. Las EVN muestran la comparación del efecto global de la media de las ocho sesiones de musicoterapia antes de iniciar las mismas y después de finalizarlas. Aún a pesar de haber obtenido unas medias de puntuaciones bajas, destaca la disminución estadísticamente significativa de los tres ítems valorados, con una mayor significación para la variable Ansiedad, en primer lugar, seguida del Malestar Físico y finalmente de la variable Tristeza. Sorprende el efecto y mejora importante del tratamiento de musicoterapia en la varia- 
ble Malestar Físico; incluso el tamaño del efecto más grande se da para esta variable también. El hecho de que el tratamiento diseñado no ha ido dirigido especialmente a trabajar este aspecto, lleva a reflexionar sobre la posible interconexión y mutua influencia de los aspectos psicológicos sobre los físicos, y viceversa. Sarafino ${ }^{(49)}$ señala la importancia de las interacciones biopsicosociales, de modo que el efecto de distractor de la música, entre otros, puede reducir la percepción de síntomas físicos y reducir consecuentemente ese malestar físico. Este ítem puede considerarse como una variable que, aunque no incide de manera directa en el estado de ánimo, sí que puede modularlo e influenciarlo.

Así pues, en base a estos resultados se puede afirmar que las sesiones de musicoterapia han resultado ser efectivas para los participantes en reducir los niveles de percepción de su propio distrés emocional relacionado con la Ansiedad, la Tristeza, y el Malestar Físico.

En lo que respecta a la variable Calidad de Vida, los resultados de la escala EORTC QLQ-C30 no muestran cambios significativos para la Escala de Calidad de Vida Global, aunque debe decirse que los participantes ya presentan unos niveles medio-altos antes de iniciar el tratamiento, lo que representaría una calidad de vida media-alta. Con respecto a las Escalas de Funcionamiento, si bien no se han obtenido cambios a nivel de significación estadística para ninguna de sus 5 dimensiones, sí debe destacarse el hecho de que la subescala de Funcionamiento Emocional es la que presenta una disminución más importante. Ello sería congruente con los objetivos del estudio, en tanto que es la dimensión sobre la que más directamente se ha pretendido incidir. Así pues, pese a que los resultados no muestran cambios significativos, el aumento en las puntuaciones en estas dimensiones permite creer que el tratamiento ha ayudado a obtener un cambio en la dirección deseada. Estos datos son también congruentes con los resultados de la revisión Cochrane ${ }^{(15)}$ que muestran una mejora en la variable calidad de vida, si bien sin diferencias significativas. Analizando la subescala de Funcionamiento Emocional, se observa como esta dimensión es la que presenta puntuaciones con cambios más notables en comparación con las otras subescalas de funcionamiento, destacando el hecho de que los participantes inician el tratamiento a un nivel de Funcionamiento Emocional con una media de puntuaciones más baja que en cualquiera de las otras cuatro dimensiones, y es precisamente aquella que muestra un cambio más notable tras la intervención de musicoterapia. Se trata de un dato interesante, que nos permite hablar sobre la capacidad de la musicoterapia para disminuir el malestar emocional y mejorar la calidad de vida.

La variable calidad de vida es un constructo multidimensional, constituido a su vez por otras dimensiones o factores ${ }^{(44)}$. Dicha variable, además de ser evaluada mediante instrumentos específicamente diseñados para medirla, puede ser también sensible a otras variables relacionadas con aspectos físicos (dolor, relajación, tensión, etc.) y/o psicológicos (ansiedad, tristeza,...), que están interconectados y que se ven mutuamente influenciados. Las intervenciones musicoterapéuticas pueden tener una influencia en los procesos fisiológicos y psicológicos, y pueden contribuir a una disminución tanto del malestar físico como del distrés emocional ${ }^{(50-52)}$.

El análisis cualititativo se realiza a partir de la información recogida mediante el Cuestionario de Valoración del Programa de Musicoterapia (CVPMT), diseñado ad hoc. Autores como Serra ${ }^{(35)}$ o Waldon ${ }^{(46)}$, complementan su análisis cuantitativo con el cualitativo, obteniendo información adicional muy interesante. El CVPMT ha permitido recoger también un tipo de información 
no sólo interesante sino también muy útil, en tanto que aporta una serie de descripciones realizadas por los participantes sobre su experiencia vivida en este programa y que podrían relacionarse también con su calidad de vida. Algunas de ellas son de carácter más general y consideran que este programa ha sido: "Muy positivo" y "Bueno"; otras expresan sentir su "Contento" con él y haber resultado de ayuda en su mejora del estado anímico: "Satisfactoria, alegría y ayuda personal", "Me ha ayudado a ser más feliz", "Tranquilizador".

Otras descripciones se centran más en el modo en que les ha sido de utilidad así como en el aprendizaje de recursos de auto-cuidado para la mejora del estado de ánimo, y tienen que ver con algunas de las estrategias de autorregulación emocional y los objetivos terapéuticos trabajados durante las sesiones refiriéndose a la experiencia como: "Me ha dado un estado de paz interior excelente", "Distraer la mente", "Muy interesante, ameno, educativo", "Me ha ayudado mucho a evadirme y relajarme y pensar en positivo en los momentos en que me venía abajo". Ha habido descripciones que tienen que ver con los aspectos grupales y el carácter terapéutico y de ayuda del mismo. Cabe recordar que diez participantes valoran en "Mucho" el grado de ayuda del programa para promover la socialización y relación entre compañeros, y los otros 8 lo valoran como "Bastante". Algunas de estas descripciones son las siguientes: "Me ha gustado mucho que hable uno y otro de la enfermedad", "Te sientes arropada y comprendida por personas que tienen tu mismo problema", "Relacionarme con otras personas que sufren el mismo problema y hacer amistad con ellas", "Sirve de ayuda para las personas que se encuentran en esta situación, porque comunicas emociones y fortaleces el ánimo para "hacer camino". Todo ello nos lleva a entender que todos los participantes consideren este programa como muy o bastante recomendable, y que su percepción del grado de utilidad del mismo sea también muy alta para la mayoría de ellos, bastante para algunos otros, y tan solo poco útil en el caso de una persona.

Así pues, y en lo que respecta a la batería de tests estandarizados administrados en este estudio, se puede ver cómo -a excepción de las EVN- todos ellos presentan unos resultados que pese a representar una mejora emocional no ofrecen un cambio a nivel estadísticamente significativo. Este hecho nos lleva a reflexionar sobre tres aspectos: la importancia de aumentar la muestra de sujetos participantes, de incluir a sujetos con un mayor nivel de malestar emocional, y de seleccionar aquellos tests estandarizados más adecuados a las variables de medición y objeto de estudio. La musicoterapia no cuenta todavía con tests estandarizados propios de su disciplina, y como debe apoyarse en aquellos que provienen de otras disciplinas, que podrían no recoger ni ser suficientemente sensibles a aquellos aspectos propios de esta discipli$\mathrm{na}^{(53,54)}$. Por otra parte, existe el dilema de mantener un equilibrio entre seleccionar y administrar tests más largos, con todos los módulos y extensión correspondiente, y adecuar el tiempo de administración de los mismos al estado físico y psicológico de los pacientes.

A modo de resumen de resultados, y basándonos en la mejora de todas las puntuaciones obtenidas, tanto de aquellas estadísticamente significativas como no, nos permite hablar de una mejora del estado anímico de los participantes tras finalizar su participación en este programa de musicoterapia. Es importante ser cauto con estos resultados, dado que no puede descartarse la presencia de otro tipo de variables que hayan podido afectar al estado emocional. No obstante, debe destacarse también el papel que haya podido desempeñar el tipo de diseño del tratamiento y las técnicas empleadas. La musicotera- 
pia cuenta con una variedad de técnicas de expresión emocional y comunicación, como son el análisis lírico de canciones, la composición musical, las audiciones musicales y cantar canciones; todas ellas han permitido expresar emociones y sentimientos a nivel verbal y no verbal, y canalizarlos a través de experiencias musicales.

Las técnicas que han tenido mejor acogida en este grupo, y de manera mayoritaria, han sido la técnica de análisis lírico, tocar instrumentos de percusión, cantar la canción de bienvenida en grupo y la relajación acompañada de música de fondo. Las audiciones de canciones que presentan un mensaje significativo permite llevar a cabo un ejercicio de análisis y exploración de temas y aspectos no resueltos, y reflexionar sobre la propia manera de pensar, sentir y actuar, así como observar otras estrategias y maneras de afrontamiento de las compañeras que pueden ser diferentes. Constituyen, pues, una interesante y eficaz estrategia para fomentar la ventilación y expresión emocional. La técnica de tocar instrumentos musicales de pequeña percusión ayuda también a canalizar emociones a nivel no verbal, y permite, a su vez, realizar un trabajo de focalización de la atención en la tarea musical. Las actividades de tocar instrumentos o de cantar juntos en un grupo permiten crear un espacio de mayor complicidad y sincronización entre los participantes, y ayuda a la cohesión del grupo $^{(46,55-57)}$.

También han sido bien recibidas las técnicas de relajación, especialmente la de escuchar música de fondo acompañada de las pautas verbales de la musicoterapeuta. Ello ha permitido canalizar la tensión muscular y/o dolor, y conseguir así distraer y liberar el malestar físico. Las distintas actividades de relajación aplicadas han generado un espacio más personal e íntimo durante las sesiones, en el que se favorece más el contacto con uno mismo, pese a estar en contexto grupal. Paralelamente, otra estrategia para promover estados de distensión y disminución de la percepción del dolor o malestar físico ha sido precisamente la utilización de técnicas de carácter activo como tocar instrumentos y música y movimiento.

Las respuestas individuales a la música son diferentes según cada persona. Este programa ha seguido la recomendación de la revisión Cochrane ${ }^{(15)}$ de utilizar no solo técnicas pasivo-receptivas sino también técnicas activas a fin de poder trabajar adecuadamente las necesidades del paciente oncológico y los correspondientes objetivos terapéuticos. Ello ha contribuido a facilitar una mayor implicación y participación. Las anotaciones recogidas en la Hoja de Observación y Registro de Conductas indican que, si bien, en los momentos iniciales de la intervención, los participantes mostraban cierta reticencia ante propuestas musicoterapéuticas más interactivas y de mayor grado de implicación, esta reticencia e inseguridad se ha ido reduciendo progresivamente, y los pacientes han ido mostrando mayor seguridad y disfrute ante las mismas.

El CVPMT revela el alto grado de utilidad del programa que han percibido los asistentes. La auto-administración de las EVN antes y después de cada sesión ofrece la oportunidad de conectar con uno mismo, de observar el propio estado emocional y físico de ese momento, y de tomar consciencia de los cambios que se puedan producir al finalizar la sesión. Pese a que la media de estas puntuaciones observadas a nivel de pre-sesión no haya sido en general demasiado alta, probablemente les haya ayudado a mejorar su insight, y a darse cuenta de que el hecho de implicarse activamente en una experiencia de este tipo puede ayudar a aumentar su sensación de control, y a tomar decisiones orientadas hacia el cuidado de sí mismos. Este estudio va en la línea de aportar una modalidad de intervención que tiene en cuenta las 
necesidades de las personas con CCR, y les ofrece nuevos recursos que les ayuden a adaptarse en su día a día.

Un aspecto muy importante a comentar tiene que ver con el porcentaje de asistencia a las sesiones así como con la adherencia al tratamiento de musicoterapia. Llama la atención el alto porcentaje de sujetos que se ha perdido en el estudio: $56,09 \%$, una pérdida de sujetos que se ha producido en dos momentos temporales del programa. Un primer momento corresponde al proceso de derivación, momento en el que los oncólogos informan del programa a sus pacientes y les recomiendan participar en él. De los 41 pacientes que fueron invitados a participar en el estudio, 16 de ellos no llegaron a hacerlo y no asistieron a ninguna sesión. Algunos de los motivos que han llevado a esta falta de adherencia al programa tiene que ver con cambios en la dinámica del día a día (problemas familiares, solapamiento del horario con otros tratamientos médicos, desconfianza y/o desconocimiento sobre la utilidad del programa, prejuicios y miedos por "no tener estudios musicales", etc.). La musicoterapia es todavía una disciplina emergente en nuestro país, y la población puede no estar todavía informada ni convencida de sus posibles beneficios. Iniciar un tratamiento nuevo, desconocido, puede resultar difícil de asumir en un momento en el que el paciente ya se suele sentir abrumado por los tratamientos oncológicos activos, las diferentes pruebas y controles médicos, así como con malestar físico y emocional. Por esta razón, sería interesante agilizar el proceso de derivación así como las listas de espera del programa y configuración de los grupos, a fin de mantener la motivación generada por los oncólogos de referencia e iniciar los grupos lo antes posible.

Un segundo momento temporal tiene que ver con el periodo de tratamiento en sí. Las dificultades de adherencia no solo a los tratamientos médicos sino también a tratamientos coadyuvantes como el soporte psicológico, entre otros, constituyen un problema frecuente en el contexto oncológico $^{(58,59)}$. En el campo de la musicoterapia sucede algo similar, y tampoco resulta fácil conseguir un porcentaje elevado de asistencia y adherencia a las sesiones, dado que existen diferentes factores que interfieren y pueden dificultar, e incluso impedir, la asistencia del paciente a la sesión: solapamiento de visitas con el oncólogo y/o otros profesionales, analíticas y/o otras pruebas médicas, etc. así como malestar físico derivado de los tratamientos, complicaciones y/o progresión de la enfermedad, o incluso éxitus del paciente. De los 25 sujetos que participaron en este estudio, 7 de ellos fueron excluidos del análisis estadístico del estudio por no asistir a un mínimo del $50 \%$ de las sesiones y uno por no haber realizado los post-tests, quedando así una muestra final de 18 participantes. Aumentar la frecuencia de las sesiones y ofrecer un programa de 2 sesiones semanales facilitaría recibir al menos una sesión por semana. No obstante, éste es un problema muy frecuente en el contexto de la musicoterapia, al que varios autores han hecho ya referencia ${ }^{(14,30,32,35)}$. Pese a todo ello, la media de asistencia ha sido de 6,16 sesiones de las 8 en total, y su correspondiente porcentaje del 77,08 , una cifra notable teniendo en cuenta que 11 de los 18 sujetos (un $61,11 \%$ ) se encontraban en ese momento recibiendo tratamiento de quimioterapia, y por tanto, podían estar experimentando los efectos colaterales de dicho tratamiento.

Los autores son conscientes de que una de las limitaciones de este estudio es el tamaño pequeño de la muestra, el carecer de una muestra aleatorizada, y no haber contado con un grupo control. Razones prácticas han tenido que ver con 
este hecho: se trata de un proyecto piloto, que no sólo pretende estudiar el efecto de la musicoterapia en los pacientes, sino también analizar cómo este tipo de programa puede incluirse progresivamente en los servicios del hospital. Es por ello que la muestra fue totalmente asignada al grupo experimental, ayudando a una coordinación más fácil entre los oncólogos y la musicoterapeuta. La muestra presenta también problemas de heterogeneidad, que pueden ser atribuidos al proceso de derivación y a unos criterios de inclusión demasiado amplios. De cara a futuros estudios, se recomienda acotar más las variables clínicas referentes al tipo de tratamiento actual así como tratamientos recibidos previamente al iniciar el programa de musicoterapia, y conviene también reflexionar sobre establecer como criterio de inclusión a aquellos sujetos que presentan un mayor grado de malestar emocional en cuanto a nivel de ansiedad y depresión, por las razones expuestas anteriormente. Además, un grupo más homogéneo en cuanto a las variables sociodemográficas permitiría abordar determinadas cuestiones y preocupaciones en la sesión de una manera más directa y todavía más adaptada a las necesidades de cada participante. Con respecto a las ganancias terapéuticas logradas, sería importante poder realizar un seguimiento de los participantes, a fin de detectar si los cambios observados en el estado de ánimo se mantienen a lo largo del tiempo.

Por otro lado, y también en relación a la muestra, cabe destacar la importancia de haber diseñado y centrado este estudio en un grupo de pacientes exclusivamente diagnosticados de cáncer de tipo colorrectal. Cada cáncer conlleva un tratamiento distinto, comporta unos efectos secundarios diferentes así como unas secuelas específicas. Desde el punto de vista clínico, el enfermo de CCR debe realizar unas adaptaciones concretas fruto de su tipo de cáncer y de sus tratamientos específicos. Desde el punto de vista terapéutiCO, trabajar con un grupo homogéneo en cuanto a diagnóstico ha facilitado la cohesión grupal, promoviendo un nivel de complicidad fuerte entre los participantes. Además, de manera espontánea, y ya desde las primeras sesiones, se han establecido vínculos y redes sociales fuera de la sesión. Durante la sesión se ha podido observar una alta disposición de ayuda y cooperación entre los participantes, compartiendo sugerencias, consejos y trucos personales de afrontamiento y adaptación a los retos que el CCR plantea. Los aspectos abordados en la sesión han tenido que ver con aspectos generales que se abordan en otros programas de musicoterapia oncológica en general, si bien, han ido específicamente relacionados con particularidades de este tipo de cáncer. Dentro de los generales, se puede decir que asistir y participar en sesiones de musicoterapia ha dado oportunidades para expresarse, canalizar emociones y mejorar el estado de ánimo, disminuyendo el nivel de alteración del mismo. Ha permitido abordar el miedo a los tratamientos, complicaciones de la enfermedad, dolor, muerte y desfiguración. También aspectos relacionados con la incomprensión a nivel social; $y$ ha ofrecido oportunidades para disminuir las alteraciones en el nivel de actividad social, dado que el hecho de asistir a sesiones de musicoterapia da oportunidades para disminuir el aislamiento, salir de casa y relacionarse con otros. Dentro de los aspectos específicos del CCR, se han abordado las alteraciones en la imagen corporal y autoestima: asistir a estas sesiones ha dado oportunidades para mostrarse ante los demás, y para compartir dificultades y frustraciones con respecto a los cambios sufridos a nivel físico-corporal y psicológico. El temor a la pérdida de control ha sido un tema frecuentemente discutido en las sesiones: 
asistir a este grupo ha permitido explorar y moverse en un entorno fuera de casa, adaptarse y manejar situaciones personales en un contexto terapéutico, que aporta seguridad, confidencialidad y confianza; todo ello con el fin de facilitar posteriores exposiciones a otros entornos y contextos cotidianos ya no terapéuticos.

Otro punto fuerte de este programa es el haber incluido la figura de un musicoterapeuta profesional, ya que ha permitido diseñar y adaptar las intervenciones al perfil y necesidades de las participantes, ha contemplado las preferencias musicales de cada una de ellos, y ha incorporado técnicas de musicoterapia de tipo activo y receptivo, en un formato de grupo que ha ayudado a abordar aspectos sociales y relacionales de los participantes. Por otra parte, si se revisan las experiencias realizadas con pacientes oncológicos, muchas de ellas se basan en intervenciones de una sesión o dos; pocas de ellas ofrecen una continuidad de ocho sesiones semanales de 90 minutos de duración. El efecto de la intervención de musicoterapia ha sido cuidado mediante el criterio de únicamente incluir en el estudio a aquellos sujetos que hayan asistido al menos al 50\% de las sesiones; este es un aspecto que no queda claro ni suficientemente recogido en los estudios de musicoterapia realizados en este campo. Finalmente, destacar el hecho de haber podido implementar el tratamiento en el propio hospital de referencia de los pacientes y ofrecerles una experiencia no invasiva, agradable $y$ gratificante, en un entorno que, hasta ese momento, ellos consideraban como "difícil e invasivo".

\section{CONCLUSIONES}

Los resultados de este estudio sugieren que la musicoterapia puede facilitar un cambio positivo en el estado de ánimo de los pacientes diagnosticados de cáncer co- lorrectal. Se ha podido observar una clara disminución del malestar emocional después de las sesiones. Es importante recoger una muestra más amplia y que presente mayor malestar emocional detectado por tests estandarizados a fin de potenciar el efecto del tratamiento a nivel significativo. Los participantes han percibido esta experiencia como útil, recomendable y de ayuda para mejorar su estado anímico en el día a día, y parece que les ha permitido mejorar diferentes aspectos a lo largo del programa, que pueden relacionarse con la variable calidad de vida. Es necesario seguir investigando en el campo de la musicoterapia a fin de poder seguir mejorando las limitaciones de este estudio, documentando nuevos hallazgos y contribuyendo a mejorar el estado de ánimo y la calidad de vida de los pacientes diagnosticados de cáncer colorrectal.

\section{AGRADECIMIENTOS:}

Este proyecto ha sido posible gracias al apoyo de Oncolliga - Fundació Lliga Catalana d'Ajuda Oncològica, Barcelona. Agradecemos al Dr. Páez y a la Dra. Tobeña así como al Servicio de Oncología del Hospital de la Santa Creu i Sant Pau de Barcelona su contribución en el desarrollo de este proyecto piloto. Nuestro agradecimiento también a Mar Martínez de la Universitat de Barcelona, por su asesoramiento en materia estadística. Por último, dar las gracias a cada uno de los participantes de este estudio, por su confianza e implicación en el programa.

\section{REFERENCIAS BIBLIOGRÁFICAS}

1. Sociedad Española de Oncología Médica. Las Cifras del Cáncer en España 2014. 2014; Recuperado a partir de: http:// www.seom.org/en/prensa/el-cancer-enespanyacom/104582-el-cancer-en-espana-2014 
2. National Comprehensive Cancer Network, NCCN (2011). Evidence-Based Cancer Guidelines, Oncology Drug Compendium, Oncology Continuing Medical Education [Acceso 20 de octubre de 2015]. Disponible en: ://www. nccn.org/

3. Hernández M., Cruzado JA. La atención psicológica a pacientes con cáncer: de la evaluación al tratamiento. Clínica y Salud 2013;24:1-9. Doi: http://dx.doi. org/10.5093/cl2013a

4. Pocino M, Luna G, Canelones P, Mendoza A, Romero G, Palacios LE, et al. La relevancia de la intervención psicosocial en pacientes con cáncer de mama. Psicooncología 2007;4:59-73. Doi:

5. Yélamos C, Fernández B. Necesidades emocionales en el paciente con cáncer. En: Astudillo W, Montiano E, SalinasMartín A, Díaz-Albo E, Mendinueta C, editores. Manejo del cáncer en atención primaria. San Sebastián: Sociedad Vasca de Cuidados Paliativos; 2009:267-84.

6. Die Trill M. Impacto Psicosocial del Cancer Colorrectal. Contigo. 2007; septiembre(12):19-21.

7. Alvarado-Aguilar S, Guerra-Cruz HG, Cupil-Rodríguez AL, Calderillo-Ruiz G, Oñate-Ocaña LF. Adaptación psicosocial en pacientes con cáncer colorrectal en quimioterapia o quimiorradioterapia. Cir Cir 2011;79:439-46.

8. Molina R, Feliu J, San-José B, López JL, Martín JI, Martínez-Amores B, et al. La reinserción laboral en los pacientes con cáncer colorrectal. Psicooncología 2008;5:83-92.

9. Blanco I, Cabrera E, Llort G. Cáncer colorrectal hereditario. Psicooncología 2005;2:213-28.

10. López-Santiago S, Cruzado J, Custodio A, Feliu J. Variables asociadas al deterioro cognitivo en pacientes de cáncer de colon. Psicooncología 2011;8:301-14.

11. Soriano M, Cantero R, Olivares ME, Cruzado JA, Delgado I, Balibrea JM, et al.
Efectos de la cirugía laparoscópica en pacientes de cáncer colorrectal: calidad de vida, estado emocional y satisfacción. Psicooncología 2007;4:121-32

12. Soriano M, Blasco J, Izquierdo F, Guerra M. Herramientas de ayuda a la toma de decisiones en cáncer colorrectal: una revisión. Psicooncología 2010;7:329-39.

13. Cardoso A, Blasco T, Fernández-Castro J. Is there a relationship between positive affect and other dimensions of quality of life in colorectal cancer patients? An Psicol 2015;31:404-13. Doi:10.6018/analesps.31.2.160331

14. Hanser SB. Music therapy in adult oncology: research issues. J Soc Integr Oncol 2006;62-6.

15. Bradt J, Dileo C, Grocke D, Magill L. Music interventions for improving psychological and physical outcomes in cancer patients (Review). Cochrane database Syst Rev 2011;(8):9-11. Doi: 10.1002/14651858. CD006911.pub2.Copyright

16. Deng GE, Cassileth BR, Cohen L, Jyothirmai $\mathrm{G}$, Johnstone PAS, Kumar $\mathrm{N}$, et al. Integrative oncology practice guidelines. J Soc Integr Oncol 2007;5:65-84

17. National Center for Complementary and Alternative Medicine, CAM (2015). [Acceso 10 de octubre de 2015]. Disponible en: http://www.nlm.nih.gov/medlineplus/ complementaryandalternativemedicine. html

18. Sociedad Española de Oncología Médica, SEOM. Musicoterapia. [Acceso $10 \mathrm{de}$ octubre de 2015]. Disponible en: http:// oncosaludable.es/es/inicio/terapias-integrativas/terapia-cuerpo-mente/114216musicoterapia

19. Bechtold ML, Puli SR, Othman MO, Bartalos CR, Marshall JB, Roy PK. Effect of music on patients undergoing colonoscopy: A meta-analysis of randomized controlled trials. Dig Dis Sci 2009;54:19-24. Doi:10.1007/s10620-008-0312-0

20. Tam WWS, Wong ELY, Twinn SF. Effect of music on procedure time and sedation 
during colonoscopy: A meta-analysis. World J Gastroenterol 2008;14:5336-43. Doi:10.3748/wjg.14.5336

21. Rudin D, Kiss A, Wetz R V, Sottile VM. Music in the endoscopy suite: A meta-analysis of randomized controlled studies. Endoscopy 2007;39:507-10.

22. Clark M, Isaacks-Downton G, Wells N, Redlin-Frazier S, Eck C, Hepworth JT, et al. Use of preferred music to reduce emotional distress and symptom activity during radiation therapy. J Music Ther 2006;43:247-65.

23. O'Callaghan C, Sproston M, Wilkinson K, Willis D, Milner A, Grocke D, et al. Effect of self-selected music on adults' anxiety and subjective experiences during initial radiotherapy treatment: A randomised controlled trial and qualitative research. J Med Imaging Radiat Oncol 2012;56:473-7. Doi:10.1111/j.17549485.2012.02395.x

24. Smith M, Casey L, Johnson D, Gwede C, Riggin OZ. Music as a therapeutic intervention for anxiety in patients receiving radiation therapy. Oncol Nurs Forum 2001;28:855-62.

25. Zhao PT, Liang J, Shao QJ, Liang F, Yuan $H Q$, You FS. Interventional effects of musical therapy to physiological and psychological conditions in process of radiotherapy for patients with cancer. Chinese J Cancer Prev Treat 2008;15:1097-9.

26. Bulfone T, Quattrin R, Zanotti R, Regattin $L$, Brusaferro S. Effectiveness of music therapy for anxiety reduction in women with breast cancer in chemotherapy treatment. Holist Nurs Pract 2009;23:238-42. Doi:10.1097/HNP.0b013e3181aeceee.

27. Ezzone S, Baker C, Rosselet R, Terepka E. Music as an adjunct to antiemetic therapy. Oncol Nurs Forum 1998;25:1551-6.

28. Ferrer AJ. The effect of live music on decreasing anxiety in patients undergoing chemotherapy treatment. J Music Ther 2007;44:242-55.

29. Frank JM. The effects of music therapy and guided visual imagery on chemothera- py induced nausea and vomiting. Oncol Nurs Forum 1985;12:47-52.

30. Gimeno MM. The Effect of music and imagery to induce relaxation and reduce nausea and emesis in cancer patients undergoing chemotherapy treatment [dissertation]. University of the Pacific, 2008.

31. Sabo CE, Michael SR. The influence of personal message with music on anxiety and side effects associated with chemotherapy. Cancer Nurs 1996;19:283-9.

32. Standley JM. Clinical application of music and chemotherapy: The effects on nausea and emesis. Music Ther Perspect 1992;10:27-35.

33. Straw GW. The use of guided imagery and relaxation for the quality of life of cancer patients undergoing chemotherapy. (Master's thesis). Lakehead University. Ontario, Canada; 1991.

34. Bozcuk H, Artac M, Kara A, Ozdogan M, Sualp Y, Topcu Z, et al. Does music exposure during chemotherapy improve quality of life in early breast cancer patients? A pilot study. Med Sci Monit 2006;12:CR200-5.

35. Serra M. Comparación del efecto de una intervención con musicoterapia para mujeres con cáncer de mama durante la sesión de quimioterapia: un análisis cuantitativo y cualitativo [tesis doctoral] Barcelona: Universitat Ramon Llull; 2013. [Acceso 10 de octubre de 2015]. Disponible en: http://www.tdx.cat/handle/10803/108962

36. Xie Z, Wang G, Yin Z, Liao S, Lin J, Yu $Z$, et al. Effect of music therapy and inner image relaxation on quality of life in cancer patients receiving chemotherapy. Chinese Ment Health J 2001;15:176-8.

37. Hanser SB, Bauer-Wu S, Kubicek L, Healey M, Manola J, Hernandez M, et al. Effects of a music therapy intervention on quality of life and distress in women with metastatic breast cancer. J Soc Integr Oncol 2006;4:116-24. 
38. Bradt J, Dileo C. Music therapy for end-oflife care. Cochrane database Syst Rev 2010; (1):CD007169. Doi: 10.1002/14651858. CD006911.pub2.Copyright

39. McNair DM, Lorr M, Droppelman LF. EDITS manual for the Profile of Mood States. San Diego, CA: Educational \& Industrial Testing Service; 1992.

40. Fuentes Y, García Merita M, Melià JL, Balaguer Y. Formas paralelas de la adaptación valenciana del Pefil de Estados de Ánimo (POMS). IV Congreso de Evaluación Psicológica, 21-24 de Septiembre. Santiago de Compostela. España; 1994.

41. Zigmond AS, Snaith RP. The hospital anxiety and depression scale. Acta Psychiatr Scand 1983;67:361-70. Doi: 10.1111/j.1600-0447.1983.tb09716.x

42. Caro I, Ibáñez E. La Escala Hospitalaria de Ansiedad y Depresión. Su utilidad práctica en Psicología de la Salud. Bol Psicol 1992;36:43-69.

43. Aaronson NK, Ahmedzai S, Bergman B, Bullinger M, Cull A, Duez NJ, et al. The European Organization for Research and Treatment of Cancer QLQ-C30: A qualityof-life instrument for use in international clinical trials in oncology. J Natl Cancer Inst 1993;85:365-76.

44. Arrarás JJ, Arias de la Vega F, Illarramendi JJ, Manterola A, Salgado E, Domínguez $M A$, et al. Calidad de vida relacionada con la salud en los servicios de Oncología del Hospital de Navarra. Grupo de Calidad de Vida de la EORTC. An Sist Sanit Navarra 2011;34:9-20.

45. Arraras JI, Suárez J, Arias de la Vega F, Vera R, Asín G, Arrazubi V, et al. The EORTC Quality of Life questionnaire for patients with colorectal cancer: EORTC QLQ-CR29 validation study for Spanish patients. Clin Transl Oncol 2011;13:50-6. Doi: 10.1007/s12094-011-0616-y

46. Waldon EG. The effects of group music therapy on mood states and cohesiveness in adult oncology patients. J Music Ther 2001;38:212-38.
47. Cassileth BR, Vickers AJ, Magill LA. Music therapy for mood disturbance during hospitalization for autologous stem cell transplantation: A randomized controIled trial. Cancer 2003;98:2723-9. Doi: 10.1002/cncr. 11842

48. Martí P, Mercadal-Brotons M, Solé, C. Efecto de la musicoterapia en el estado anímico y calidad de vida de un grupo de mujeres supervivientes de cáncer de mama. Psicooncología 2015;12:105-128. Doi: 10.5209/rev_PSIC.2015.v12.n1.48907

49. Sarafino EP. Health Psychology: Biopsychosocial Interactions. 5th ed. New York: John Wiley and Sons; 2008.

50. Huang S-T, Good M, Zauszniewski JA. The effectiveness of music in relieving pain in cancer patients: A randomized controlled trial. Int J Nurs Stud 2010;47:1354-62. Doi: 10.1016/j.ijnurstu.2010.03.008

51. Magill-Levreault L. Music therapy in pain and symptom management. J Palliat Care 1993;9:42-8.

52. Zimmerman L, Pozehl B, Duncan K, Schmitz R. Effects of music in patients who had chronic cancer pain. West J Nurs Res 1989;11:298-309.

53. Dileo C. Effects of music and music therapy on medical patients: A meta-analysis of the research and implications for the future. J Soc Integr Oncol 2006;4:67-70.

54. Standley JM. Investigación sobre música en el tatamiento médico. En: Martí P, Mercadal-Brotons $M$, editores. Musicoterapia en Medicina Aplicaciones prácticas. Barcelona: Editorial Médica Jims.; 2010. p. 1-63.

55. Dvorak A. Music therapy support groups for cancer patients and caregivers [dissertation] University of lowa; 2011.

56. Carballo P, Martí P. Musicoterapia y Oncología: Una experiencia con un grupo de mujeres con cáncer de mama. Música, Terapia y Comunicación Rev Musicoterapia 2008;28:27-42.

57. Martí P, Carballo P. Musicoterapia y cáncer de mama. En: Martí P, Mercadal-Brotons $M$, editores. Musicoterapia en Me- 
dicina Aplicaciones prácticas. Barcelona: Editorial Médica Jims. 2010. p.251-70.

58. Arrighi E, Jovell AJ, Navarro MD. El valor terapéutico en oncología. La perspectiva de pacientes, familiares y profesionales. Psicooncología 2010;7: 363-74.
59. Hernández M, Cruzado JA, Prado C, Rodríguez E, Hernández C, González MÁ, et al. Salud mental y malestar emocional en pacientes con cáncer. Psicooncología 2012;9:233-57. Doi:10.5209/rev_ PSIC.2013.v9.n2-3.40895 